Sociologie et sociétés

\title{
Fragmentation et exclusion : la question sociale dans la phase actuelle de la transition des villes dans les sociétés industrielles avancées \\ Fragmentation and Exclusion: The Social Question in the Present Transition of Advanced Industrial Cities
}

\section{Enzo MINGIONE}

Volume 30, numéro 1, printemps 1998

Le second souffle de la sociologie

URI : https://id.erudit.org/iderudit/001507ar

DOI : https://doi.org/10.7202/001507ar

Aller au sommaire du numéro

Éditeur(s)

Les Presses de l'Université de Montréal

ISSN

0038-030X (imprimé)

1492-1375 (numérique)

Découvrir la revue

Citer cet article

MINGIONE, E. (1998). Fragmentation et exclusion : la question sociale dans la phase actuelle de la transition des villes dans les sociétés industrielles avancées. Sociologie et sociétés, 30(1), 69-83. https://doi.org/10.7202/001507ar

\section{Résumé de l'article}

Cet article est fondé sur une critique radicale du paradigme du marché auto-régulé et facteur de régulation sociale. On soutiendraque les relations d'échange ne peuvent avoir lieu que dans des conditions favorables que ne peut pas directement créer lemarché lui-même, mais qui sont plutôt imputables à des facteurs de coopération (association et réciprocité) constituant la basede l'enracinement social de l'économie. Ceci a deux conséquences importantes : d'une part, les différentes conditions sociales(dans le temps et dans l'espace) sont essentielles à la compréhension des modes de développement industriel ; d'autre part, les sociétés industrielles se caractérisent par des différences importantes et persistantes. L'époque fordiste a été caractérisée parun mélange de conditions où les associations étaient prédominantes tandis que l'époque actuelle se caractérise par un retourde la réciprocité ; c'est en ce sens que l'on considère que les sociétés contemporaines sont fragmentées. On fera ici une étudecomparative montrant à quel point les traits de la fragmentation se manifestent dans la production de l'exclusion sociale, c'està-dire d'un statut discriminatoire appliqué institutionnellement, venant s'ajouter à certains processus de marginalisation tenantau chômage et à la transformation des systèmes socio-démographiques et des systèmes d'emploi. 


\section{Fragmentation et exclusion :la question sociale dans la phase actuelle de transition des villes dans les sociétés industrielles avancées}

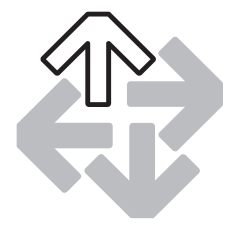

ENZO MINGIONE

(Traduction : Véronique Dassas)

\section{INTRODUCTION : QUELQUES INTERPRÉTATIONS DE LA QUESTION SOCIALE AU-DELÀ DU PARADIGME DU MARCHÉ}

À partir de 1970, certains changements profonds affectent la vie économique dans les pays industrialisés. On définit les sociétés contemporaines comme post-fordistes ou post-modernes. De plus en plus, les gens ne veulent plus entendre parler ni de la bureaucratie, ni des interventions de l'État dans l'économie, ni des grandes organisations hiérarchiques. On parle aujourd'hui de globalisation et de flexibilité, des perspectives offertes par les comportements ouvertement utilitaires dans des contextes où la régulation, libérée du poids de l'État-providence, est censée produire innovation et efficacité. Selon cette perspective, nous passons en ce moment d'un capitalisme «providentialiste » à un capitalisme global. Il s'agit d'un système moins structuré, dominé par les flux financiers et les flux d'informations où le travail des gens, la vie des familles sont instables et où la capacité de contrôle des États-nations sur leurs économies est faible. Les politiciens et les économistes qui défendent cette conception des changements actuels ne tiennent compte que des paramètres du marché et ignorent que les facteurs sociaux demeurent importants, aujourd'hui comme par le passé. Même les interprétations les plus novatrices, comme celles de Rifkin (1996) dans La Fin du travail ou encore celles de Esping-Andersen (1990) dans Three Worlds of Welfare Capitalism, sont marquées par le déterminisme des approches qui ignorent l'importance de la compatibilité entre les perspectives économiques et la question sociale : il n'y a pas d'économie qui tienne sans un niveau suffisamment élevé de mécanismes d'intégration qui alimentent le potentiel de coopération. La flexibilisation et la précarité, les technologies qui remplacent le labeur humain et les formes nouvelles de la division du travail ne peuvent stimuler la croissance économique et accroître le bien-être de la société que si elles n'entraînent pas une désagrégation sociale incontrôlable et chèrement payée, y compris en termes économiques, qui mine le potentiel de coopération. Aujourd'hui, la tâche de la sociologie est de se pencher à nouveau sur les facteurs sociaux, au-delà du paradigme du marché. Cet article essaiera d'aller dans cette direction, suivant la voie tracée à grands traits mais restée peu développée par Granovetter (1985) pour reprendre l'élaboration d'une théorie de l'enracinement social du comportement économique.

La critique du paradigme du marché a des racines dans les travaux de Polanyi (1983), où l'on trouve déjà une autre approche fondée sur l'enracinement social du comportement 
économique ${ }^{1}$. Polanyi sous-évalue le fait suivant : si l'existence de formes d'organisation repose sur des principes de coopération, le concept pur d'interaction du marché construit sur l'idée de compétition utilitariste atomisée ne correspond pas à des formes d'organisation, mais au contraire présuppose l'absence d'organisation ${ }^{2}$. Cependant, un grand nombre d'échanges marchands systématiques, caractéristiques des sociétés industrielles, ne peuvent se situer en dehors de contextes de coopération. On doit supposer que les sociétés de l'ère industrielle sont fondées sur un mélange de tensions déstructurantes, dues au marché, et de principes de coopération qui ne sont pas directement produits par lui ${ }^{3}$. Une relecture sociologique de l'approche anthropologique de Polanyi permet de repérer ces principes, comme l'association - le partage de mêmes intérêts personnels — et la réciprocité — l'appartenance à un groupe restreint où les buts communs l'emportent sur les intérêts individuels immédiats. L'approche fondée sur l'enracinement reflète le fait que les sociétés individualisées de l'âge industriel sont construites autour de combinaisons contextuelles de tensions engendrées par l'accroissement des perspectives offertes par le marché en termes de compétition utilitariste et du jeu changeant des réponses de la coopération sociale fondées sur un mélange d'association et de réciprocité.

Contrairement aux approches fondées sur le paradigme du marché, le concept d'enracinement social souligne l'importance de deux facteurs : les différences provoquées par les diverses combinaisons de réponses sociales, qui se cristallisent en une pluralité de modèles et de variantes des sociétés industrielles, et le changement qualitatif historique, qui se manifeste par de longs cycles caractérisés par la prédominance de modes de combinaison entre développement économique et régimes de régulation ${ }^{4}$. De ce point de vue, pour discuter de la question sociale aujourd'hui, on doit se situer dans le cadre d'un schéma (voir figure 1) qui clarifie la variété des interactions entre les tensions du marché et les facteurs de coopération dans une double perspective : celle du développement historique et celle des différents modèles qui se sont cristallisés dans des contextes où la réponse de la socialité se caractérise systématiquement par différentes combinaisons d'association et de réciprocité.

Les sociétés contemporaines se trouvent à la fin d'un cycle historique qui a témoigné de la prévalence des facteurs organisationnels associatifs. Les principaux ingrédients de ce capital de régulation ont été la prédominance des économies d'échelle et le développement des groupes industriels, les programmes d'assistance publique (État-providence), la consommation de masse standardisée, l'institutionnalisation des groupes d'intérêts, la famille nucléaire fondée sur le système du pourvoyeur (emploi masculin à temps plein et discrimination économique systématique à l'endroit des femmes, le tout combiné aux mutations profondes des modèles de reproduction et des responsabilités assumées principalement par les femmes) et l'individualisme familial (investissement dans la mobilité intergénérationnelle ascendante de l'enfant mâle). Ces traits se sont développés de diverses façons dans les différents modèles de capitalisme «providentialiste ». Nous nous sommes inspiré, dans la figure 1, de la classification proposée par Esping-Andersen (1990) en la modifiant pour faire place à des données qualitatives sur le rôle à la fois des cycles historiques et des différences

1. Pour plus de détails théoriques sur une approche néo-polanyienne, le lecteur se reportera à d'autres travaux, notamment à ceux de GRANOVETTER (1985) et de MINGIONE (1991).

2. En ce qui concerne le marché auto-régulé, Polanyi soutient : «Une telle institution ne pouvait exister de façon suivie sans anéantir la substance humaine et naturelle de la société, sans détruire l'homme et sans transformer son milieu en désert. » (Polanyi, 1983 ; p. 22).

3. Dans le chapitre I de MINGIONE (1991), j'explique pourquoi je suis en désaccord avec la solution adoptée par Polanyi qui attribue au marché le potentiel de créer indirectement des institutions organisationnelles et de coopération. Dans mon raisonnement, j'en arrive à considérer que deux principes «purs » de coopération demeurent : l'association et la réciprocité. Naturellement, ils se transforment et se confondent sous diverses formes, en particulier là où les tensions déstructurantes deviennent plus fortes du fait de la diffusion dans le marché des possibilités de compétition rationnelle et de la progression de l'individualisme.

4. La question des cycles historiques est au cœur des travaux de Fernand BRAUDEL (1977, 1986a, 1986b) et d'Emmanuel WALLERSTEIN (1995,1986, 1996). L'hypothèse de la succession de cycles historiques organisés et désorganisés a été autoritairement avancée par l'historien Henri Pirenne (1953). Voir aussi les travaux récents de Giovanni ARRIGHI (1994). 
FIGURE 1

Cycles historiques et modèles de développement

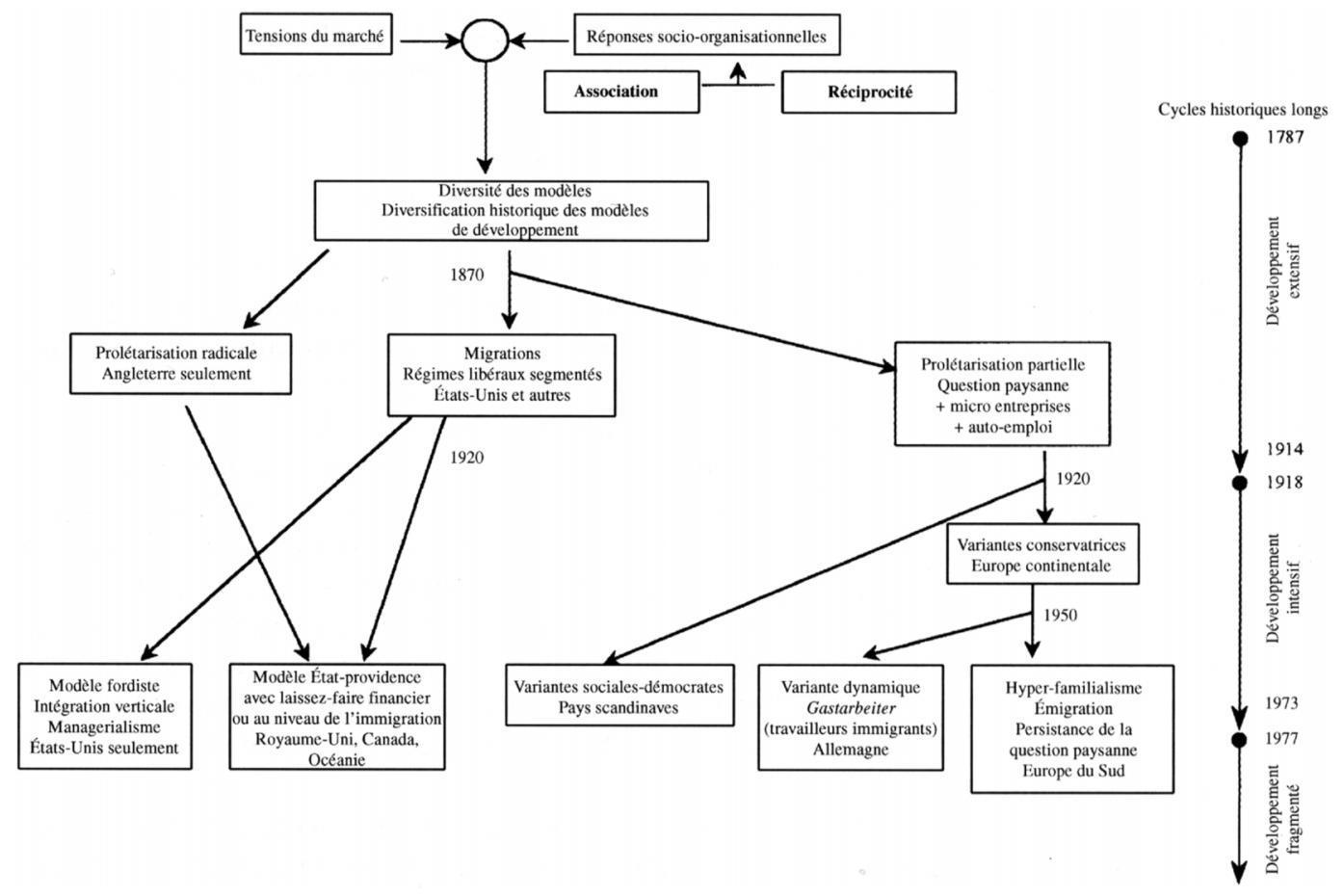

qui ont pris forme en termes de constructions systématiquement différentes des réponses sociales aux tensions du marché. Dans le premier cycle historique (extensif), les différentes voies furent les suivantes : la prolétarisation radicale (au Royaume-Uni), la persistance à long terme de la question paysanne (en Europe centrale et au Japon) et l'afflux de populations immigrantes (en Amérique du Nord et en Océanie). Au cours du cycle intensif suivant — l'époque fordiste —, les États-Unis devinrent une puissance hégémonique et ils adoptèrent un modèle unique centré sur de hauts niveaux d'intégration verticale, la gestion, le taylorisme, les compagnies privées d'assistance sociale et le dualisme entre l'économie de la majorité et celle des immigrants. Le Royaume-Uni amorça son déclin et rejoignit les autres pays d'immigration en alliant réformes de l'assistance publique et laissez-faire financier. À l'intérieur du modèle de la persistance de la paysannerie, on a pu distinguer au moins trois variantes : 1) une solide alliance consolidée entre certaines strates de la population agraire et des ouvriers pour développer les programmes d'assistance publique (le modèle social-démocrate en Scandinavie) ; 2) un degré assez haut de complémentarité entre l'intervention de l'État, l'innovation dans la petite entreprise et le rôle privilégié des familles (des femmes) dans la production d'une large part des services sociaux (modèle étatique conservateur en Europe centrale et au Japon) ; 3) une persistance plus forte des petites entreprises agricoles et des fermes, dans des formes moins dynamiques et avec une intervention de l'État plus modeste, et des vagues incessantes d'émigration (modèle familial typique surtout des pays de l'Europe du Sud).

J'essaie ici de montrer comment l'actuelle transition mène à de sérieuses difficultés du point de vue social dans tous les différents modèles, même si ces difficultés prennent des formes diverses. L'accent mis partout sur les facteurs associatifs standardisés, les grandes organisations et les divisions formelles qui ont caractérisé une phase historique centrée sur l'accroissement massif de la production dans de grandes unités industrielles, ne parvient plus à maintenir un contrôle sur une société plus fragmentée et hétérogène. À bien des égards, les sociétés contemporaines sont plus 
hétérogènes et diversifiées, ce qui reflète le regain d'importance des facteurs de réciprocité dans les comportements novateurs, depuis le rôle des réseaux économiques (Castells, 1996) jusqu'à l'utilisation des relations personnelles ou de la solidarité ethnique dans les parcours professionnels. C'est à l'intérieur de tels processus que la transition mine la traditionnelle cohésion fordiste entre perspectives économiques et capacités de coopération et, par conséquent, active de diverses façons les tendances à la désintégration sociale et à l'exclusion.

\section{LA TRANSITION ACTUELLE DES RÉGIMES D’EMPLOI}

Dans tous les pays industrialisés, la régulation sociale des régimes d'emploi est mise en péril par de sérieux problèmes qui se manifestent par l'augmentation du chômage à long terme ou la généralisation des emplois précaires rapportant des revenus insuffisants, ce qui a pour effet d'accroître les inégalités sociales et la pauvreté chronique. Les principales causes de cet état de fait seraient la globalisation et la restructuration industrielle ; la transition démographique (Lesthaeghe, 1991) et le vieillissement de la population ; l'augmentation des dépenses publiques pour financer des systèmes d'assistance sociale de plus en plus coûteux (Esping-Andersen, 1996). Mais ces causes ne sont en fait que les effets des combinaisons variées des tensions du marché et de la régulation nécessaire afin que les régimes d'emplois salariés puissent exister dans une indispensable structure d'organisation sociale. Les formes de sélection, de division et de protection de l'offre d'emploi qui accompagnèrent l'intensification du travail pour atteindre des taux de productivité élevés dans le cadre des systèmes d'État-providence, de plein emploi masculin et de la formation de la famille nucléaire vivant sur le salaire d'un pourvoyeur ne sont plus adéquates.

Les systèmes d'emploi ont été frappés par divers changements, tous centrés autour d'un mélange de désindustrialisation - le déclin de l'emploi en usine surtout dans les grands groupes industriels — et la tertiairisation — l'augmentation de l'emploi dans le secteur des services, qu'ils soient publics ou privés. Suite à ces changements, l'emploi global a augmenté dans certains contextes (surtout aux États-Unis, au Japon et en Scandinavie) et a diminué dans d'autres (dans presque tous les pays d'Europe centrale et d'Europe du sud), mais, dans tous les cas, l'image de l'emploi est devenue plus hétérogène et le modèle de l'emploi permanent du pourvoyeur du revenu familial a cessé d'être prévalent d'où une redéfinition des mécanismes de régulation sociale. C'est précisément ce processus qui donne lieu à des problèmes de plus en plus importants, soit l'intensification de la fragmentation sociale et la réémergence de l'exclusion.

La désindustrialisation et la restructuration industrielle ont été les résultats à long terme des hausses de productivité qui ont caractérisé les Trente Glorieuses, après-guerre. La crise du pétrole a contribué à intensifier un processus qui est essentiellement structurel. De telles hausses massives de la productivité dans les secteurs concentrés de la production de biens de consommation durables ont des effets à long terme sur la baisse de l'emploi, car elles ne peuvent être soutenues éternellement par une croissance exponentielle de la consommation de masse. Les choses ont été compliquées par la globalisation, celle-ci ayant favorisé l'émergence de nouvelles zones de production et des formes diverses de relocalisation et de réorganisation des entreprises.

Le déclin de l'emploi industriel dans les grandes entreprises, qui précisément est à la charnière de la régulation sociale en combinant sélection et conditions de forte compétitivité à tous les niveaux de compétence de la force de travail, a eu pour effet de destabiliser les systèmes d'emploi, même si le taux d'emploi n'a pas baissé ou si le chômage n'a pas augmenté. On constate un amoindrissement de l'effet bénéfique du circuit qui maintenait l'équilibre entre les modes de régulation sociale et les augmentations de la productivité. Dans les sociétés industrielles avancées, une part croissante de la main-d'œuvre disponible qui occupe traditionnellement des emplois permanents à haut niveau de productivité et rémunérés en fonction d'un coût de la vie et d'un niveau de consommation élevés ne réussit plus à trouver des emplois adéquats. Tous les pays industriels avancés sont donc en train d'entrer dans une phase d'excédent chronique de main-d'œuvre disponible. Il ne s'agit pas d'un excédent absolu et, en fait, de nombreux emplois existent dans le secteur des services, mais 
ils sont toutefois pour la plupart trop précaires et trop mal payés pour correspondre aux aspirations des travailleurs qui pourraient s'y intéresser. On a donc un déséquilibre qui affecte les modalités mêmes de la régulation des connexions entre les formes d'emploi et les conditions sociales d'existence. Cela explique aussi pourquoi ce processus a produit divers syndromes attribués à la brutalité de la restructuration industrielle ou à la faillite des politiques économiques, alors que ni l'une ni l'autre n'ont eu d'importance décisive.

Les deux syndromes négatifs de la crise de l'équilibre régulateur des régimes d'emploi sont l'augmentation des taux de chômage et l'accroissement du nombre des emplois précaires insuffisamment payés. La première est typique de quelques pays européens, tandis que le second a son épicentre aux États-Unis. Cependant, dans tous les cas, on a un mélange de phénomènes reliés également au travail indépendant, aux systèmes d'assistance sociale et au profil des sans-emploi et des hommes qui en ont un, des facteurs qui ont différentes répercussions sur les choix de vie et sur les modalités permettant d'intégrer socialement le fait de passer de longues périodes à chercher du travail ou dans une situation d'emploi précaire.

Après les taux peu élevés du début des années 1970, le chômage a augmenté dans tous les pays industriels, avec cependant des variations sur le moment, les modalités ou les situations spécifiques ${ }^{5}$. L'augmentation a été beaucoup plus importante en Europe, liée également à un accroissement du temps d'attente et donc du chômage de longue durée. C'est surtout par rapport à ce dernier que se manifeste la vraie nature du problème, c'est-à-dire la crise des formes existantes de la régulation sociale du marché du travail. Dans presque tous les cas, le chômage prolongé affecte surtout des sujets dont le profil personnel et social ne leur permet par d'accepter des carrières qui les feraient baisser dans la hiérarchie sociale mais pour qui, en même temps, il n'y a pas d'autre choix et qui, d'une façon ou d'une autre, sont protégés par une combinaison d'assistance de l'État et d'assistance familiale. Dans les pays d'Europe du Sud, ce sont surtout des jeunes qui sont entretenus par leurs parents. En Allemagne, ce sont des adultes d'âge mûr qui sont proches de l'âge de la retraite, appartiennent surtout à des minorités immigrantes et ne sont capables de survivre que grâce aux allocations de chômage ou de salaire minimum. Presque partout en Europe, ce sont surtout les femmes qui sont au chômage, sauf au Royaume-Uni ${ }^{6}$, où elles trouvent les emplois à temps partiel, une option qui n'est pas viable pour les hommes. Récemment, les pays scandinaves, qui semblaient s'en sortir indemnes, ont été atteints à leur tour par la crise du chômage ${ }^{7}$. L'importance de la question de la régulation au-dessus et au-delà du marché y est apparue plus clairement qu'ailleurs. Les difficultés du secteur privé à absorber les nouvelles générations de travailleurs ont frappé ensuite le secteur public, qui, pour faire face à la crise financière des réductions de budgets, a été contraint de supprimer plusieurs programmes et donc d'augmenter le chômage, pris dans un mouvement de spirale difficile à enrayer.

Parce que la crise de l'emploi a ses racines dans les systèmes de régulation sociale des régimes d'emploi, il est difficile de s'attaquer au problème, d'où des erreurs. L'exemple le plus frappant, c'est celui de la qualification de la formation des gens qui sont au chômage. Étant donné que le chômage affecte surtout les sujets dont le niveau d'éducation est bas, on suppose que la principale cause du chômage, c'est le surplus de main-d'œuvre peu qualifiée (Esping-Andersen, 1996) et donc que la meilleure façon de le combattre est de relever le niveau des compétences professionnelles. En réalité, la situation est plus complexe. Les sujets peu instruits ne trouvent pas de travail ou sont obligés d'accepter des emplois peu qualifiés, ceux qui précisément disparaissent avec l'ère fordiste, alliant faible qualification et haut taux de productivité, ceux qui permettaient jusqu'ici des niveaux

5. Voir à ce sujet, entre autres, MALINVAUD (1984), D'IRIBARNE (1990), PUGLIESE (1993), BENOÎTGUILBOT ET GALLIE (1992), REYNERI (1990).

6. En Amérique du Nord et en Océanie aussi, ce sont surtout les hommes qui sont concernés par le chômage, en particulier les jeunes et les adultes d'âge mûr qui sont de trop à la suite de la désindustrialisation.

7. Le problème est d'abord apparu au Danemark, où le chômage s'est stabilisé au taux élevé de la moyenne européenne pendant les années 1980. Il s'est ensuite étendu à la Finlande, pour atteindre rapidement des niveaux très élevés. Enfin, au début des années 1990, il a atteint la Suède, montant littéralement en flèche en l'espace de quelques mois d'un taux dérisoire de 1 ou $2 \%$ aux taux moyens supérieurs se situant entre 8 et $10 \%$, où il se stabilisa. 
de vie acceptables. On ne peut pas redresser la situation en augmentant le niveau des compétences, car cela débouche presque toujours sur une course sans fin pour acquérir les qualifications minimales afin d'obtenir un emploi. Les solutions novatrices sont plutôt fondées sur la possibilité de valoriser ce qui constitue actuellement les emplois flexibles et précaires, de façon à ce qu'ils deviennent protégés socialement et acceptables pour ceux qui sont aujourd'hui au chômage ou mal payés.

Les chômeurs ne chôment pas parce qu'ils sont peu instruits; au moins, si on leur présente des alternatives réalistes, ils cherchent à éviter le piège des parcours professionnels qui ne leur permettent pas de planifier leur vie au niveau de l'intégration sociale et culturelle dans leurs milieux d'appartenance et qui mènent inexorablement à l'exclusion. C'est ce qui fait la différence entre les États-Unis et le Royaume-Uni, d'une part, et les pays d'Europe continentale de l'autre (EspingAndersen, 1996). Dans les deux premiers pays, une grande proportion des chômeurs potentiels sont forcés, faute d'alternatives, d'accepter des emplois de type précaire, ce qui a pour conséquence de faire augmenter le nombre des travailleurs pauvres. Tandis que dans les pays d'Europe continentale, la transition génère une hausse du niveau de chômage ou des dépenses de l'État. Cette transition dans le monde du travail est compliquée par le fait qu'en aucun cas on ne peut parler d'une opposition absolue entre bonnes et mauvaises situations de travail, ces dernières entraînant toujours et inexorablement les sujets à la faillite sociale et à l'exclusion. Tout dépend en grande partie du profil d'insertion socio-familiale des travailleurs les plus vulnérables.

\section{LA POLARISATION DES EXPÉRIENCES DE TRAVAIL DANS LES SERVICES}

La restructuration de l'industrie manufacturière a contribué à rendre le tableau de l'emploi plus hétérogène en réduisant le nombre des emplois garantis dans les grandes entreprises et en accroissant, dans une proportion inférieure, le nombre des emplois du secteur secondaire, dans les petites entreprises de sous-traitance, les zones de décentralisation et de relocalisation et les régions semi-périphériques du nouvel essor industriel. Néanmoins, en termes quantitatifs, ce processus de restructuration a eu des effets modestes en comparaison de l'impact déstabilisant qu'il a eu sur les systèmes de régulation sociale. Ceux-ci n'ont jamais reposé sur l'homogénéité de l'emploi industriel ou sur le fait qu'il concerne la majorité de la population active, mais plutôt sur un schéma où les conditions propres à l'emploi dans les grandes industries constituaient le paramètre clef de la régulation. Ce schéma s'appliquait selon deux conditions : un cycle d'expansion de l'emploi industriel et la capacité des conditions propres aux entreprises à haut taux de productivité industrielle d'influencer tous les types d'emplois, entraînant des ajustements au niveau des services, du travail indépendant et des micro-entreprises ${ }^{8}$. La désindustrialisation a complètement bouleversé ce portrait. Par ailleurs, les changements dans le secteur industriel concernent une proportion toujours plus petite des emplois dans les économies avancées où la majorité de la population active (plus de $60 \%$ ) est employée dans le secteur tertiaire et où presque tous les nouveaux emplois sont créés dans le secteur des services. L'hétérogénéité et la polarisation des régimes d'emplois contemporains dérivent principalement des caractéristiques du secteur tertiaire, lequel est soumis à des processus très différents de ceux qui caractérisent l'industrie manufacturière. Pour comprendre ce qui se passe aujourd'hui, il faut se pencher sur les régimes d'emploi de service (Sassen, 1991, 1996).

L'emploi dans les services est d'une importance considérable, pas seulement en fonction de sa prédominance en termes quantitatifs, mais aussi parce qu'il est caractérisé par une large variété de formes de régulation, qui se manifeste en différentes combinaisons d'activités d'assistance assumées par les familles, par les services publics ou privés. Ces combinaisons constituent un des paramètres des modèles structurants et des variantes des sociétés industrielles avancées. De plus, la variété a pour effet de diversifier la qualité de vie de différents groupes dans différents contextes

8. Prenons par exemple l'impact des « chartes des droits de travailleurs », qui furent d'abord négociées et formulées dans les grandes entreprises industrielles mais qui eurent par la suite des effets régulateurs sur presque tous les secteurs de l'emploi en établissant un standard général pour les carrières professionnelles, standard qui fit office de paramètre pour la modification d'autres emplois. 
sociaux. On peut supposer que le principal support de régulation des régimes d'emploi est en train de passer du secteur de la production industrielle au secteur des services.

Je partirai d'une interprétation économique de la dynamique typique du processus de tertiairisation pour la développer grâce à quelques commentaires sur la régulation sociale ${ }^{9}$. Ce type d'interprétation est fondé sur deux hypothèses. La première est inspirée de Ernst Engels et de sa loi de la hiérarchie de la consommation en fonction de la variation des niveaux de revenu. Selon Engels, les hausses de revenu augmentent la propension à consommer sur une échelle allant de la satisfaction des besoins de subsistance minimale aux produits de luxe et, surtout, aux services qui ne sont pas essentiels à la survie. La croissance économique et l'augmentation conséquente du revenu moyen produit une hausse plus que moyenne de la demande de services.

La deuxième hypothèse est que les différentes branches de l'économie ont des capacités variables à faire augmenter la productivité de la main-d'œuvre en introduisant des changements techniques ou organisationnels. Un large pan du secteur des services est, pour des raisons techniques, au moins en partie insensible aux hausses de la productivité de la main-d'œuvre. Les innovations techniques ou organisationnelles servent plus souvent à améliorer la qualité du service ou à le rendre disponible à la consommation de masse. Le résultat est donc un sérieux problème de productivité qui a lui-même des répercussions sur le régime d'emploi. Les services qui ont peu de potentiel de gain de productivité enregistrent des augmentations importantes des coûts et des tarifs générées par la nécessité d'augmenter les salaires des travailleurs pour qu'ils puissent continuer à rehausser leurs standards de vie au même rythme que les travailleurs marqués par une augmentation de la productivité dans d'autres secteurs de l'économie. À l'intérieur du processus de tertiairisation, la combinaison des deux facteurs dont nous avons fait l'hypothèse conduit à un cycle d'expansion alimenté d'une part par une augmentation de la demande en raison de la croissance économique et, d'autre part, par une productivité qui reste basse et qui se manifeste par une croissance de l'emploi difficile à tenir en échec avec des stratégies fondées sur l'utilisation de la technologie, puisque celleci tend à épargner des emplois et des taux de travail intensifié.

Jusqu'ici, l'explication du cycle expansif de l'emploi dans les services est plausible, mais elle ne correspond pas aux modes de régulation sociale où la main-d'œuvre est sélectionnée et où sa performance est mesurée en termes d'augmentation de la productivité. Cela n'explique donc pas pourquoi les crises sérieuses des coûts et des tarifs n'ont pas réussi à freiner ce cycle en spirale. En réalité, le processus s'accompagne de différentes combinaisons, à commencer par le développement des régimes d'assistance publique, qui explique une tenace et vertigineuse croissance et, en même temps, fait émerger de sérieuses contradictions dans les régimes de régulation. La tertiairisation crée une forte proportion de possibilités d'emplois à faible taux de productivité qui, en principe, ne sont pas compatibles en termes de salaire, de stabilité et de garanties avec le coût de la vie et les choix propres à la communauté d'appartenance et donc ne reflète pas les conditions de régulation, ce qui entraîne l'affaiblissement des mécanismes d'intégration. Jusqu'à un certain point, cette dernière contradiction n'atteint pas des degrés intolérables, justement parce que les modes de régulation la tiennent en échec par diverses combinaisons de trois processus différents : l'augmentation des services d'assistance sociale dispensés directement par l'État, l'absorption dans la famille de la demande de services ainsi que de nouvelles formes de travail non payé pour les femmes et, surtout, la généralisation de situations de travail qui ne garantissent pas à elles seules l'intégration sociale, mais où la compatibilité sociale est conférée par des profils complémentaires (détention de plusieurs emplois ; travail à temps partiel pour les femmes mariées, travail occasionnel pour les étudiants), des activités particulières ou la concordance temporaire avec les projets et les conditions de vie des travailleurs, en particulier dans le cas des immigrants ${ }^{10}$. Ce qu'il reste à comprendre, ce sont les

9. Globalement, ma ligne d'interprétation est semblable à celle de GERSHUNY $(1978,1983)$ et MILES (1983) pour expliquer l'émergence de la société du self-service.

10. Les formes de self-service qui intègrent le travail non payé de l'utilisateur (dans les restaurants, les magasins, les stations d'essence, etc.) peuvent être vues comme des homologues de ce dernier processus dans la mesure où elles ont contribué, à leur tour, à la généralisation des emplois précaires mal payés. 
conséquences sociales des diverses combinaisons et les raisons qui expliquent pourquoi toutes les combinaisons possibles ne parviennent pas à consolider suffisamment les régimes de régulation et donc créent de sérieux problèmes dans le domaine social.

Les modèles de capitalisme «providentialiste » (Esping-Andersen, 1990 ; Mingione, 1996) ont développé des combinaisons qui compressent tous les éléments du mélange. Cependant, les services publics sont prédominants de façon typique dans le modèle social-démocrate ; la prépondérance des services publics qui entraîne un accroissement des emplois incertains et mal payés caractérise le modèle du laissez-faire ; et les variantes conservatrices ont démontré une propension marquée à faire assumer une grande partie des services d'assistance au sein des familles par le travail non payé des femmes. Selon des modalités différentes, toutes les voies tendent à long terme à déstabiliser le régime social.

La voie social-démocrate est en difficulté parce qu'elle alimente un circuit de dépenses publiques à des coûts très élevés et dans des conditions relativement rigides. L'approche du laissez-faire est directement dominée par la polarisation de l'emploi qui conduit à l'extension de la pauvreté et de l'exclusion sociale chronique. C'est là que la polarisation de l'emploi est la plus évidente, tandis que dans les modèles sociaux-démocrates, elle est dissimulée par les conditions particulières de l'emploi dans les services publics et, dans le modèle conservateur, par la grande proportion de travail domestique et de travail non payé exécuté par les femmes. Ce qui, dans la voie conservatrice, mène à des tensions de plus en plus fortes, c'est que même la combinaison entre nouvelles familles et services publics de base ne peut résister au-delà d'un certain point à la tertiairisation croissante et à la fragmentation de la structure sociale qui se produit simultanément. Là encore, les problèmes viennent du coût élevé de l'assistance publique ${ }^{11}$ auquel s'ajoute le fait qu'on ne peut faire peser la responsabilité des systèmes de soin sur les familles (les femmes) au-delà d'une certaine limite, spécialement dans un contexte où les familles deviennent instables et où les besoins d'assistance et la bureaucratisation des relations avec les autorités s'accroissent.

En conclusion, les choses étant ce qu'elles sont, la polarisation des régimes d'emploi est incontrôlable même si, dans certains cas de capitalisme « providentialiste », elle est en partie cachée par des formes de fonctionnariat et dans d'autres par la persistance de travail d'assistance non payé effectué par les femmes pour leurs familles. Aucune de ces situations, cependant, ne permet la résistance des équilibres socio-régulateurs développés dans la phase de l'industrialisation à grande échelle qui a connu l'expansion des régimes d'emplois salariés dans les pays industriels avancés. Les tensions critiques n'ont pas été provoquées par la surproduction d'une main-d'œuvre peu formée mais par la prolifération de perspectives d'emploi qui ne donnent aucune garantie de stabilité, de salaire et de conditions de travail permettant une intégration complète et durable dans une communauté d'appartenance. C'est en ce sens que la crise de l'emploi s'est transformée en crise sociale.

\section{EMPLOI FLEXIBLE VERSUS POLARISATION ET FRAGMENTATION}

La restructuration et la tertiairisation posent des problèmes insurmontables par rapport aux formes de régimes sociaux de régulation qui se sont développées à l'époque du capitalisme « providentialiste ». Ces processus n'ont pas amoindri le besoin de soutenir les carrières hautement productives par des systèmes de soutien et de sélection bien pensés. Au contraire, l'augmentation de la productivité et l'arrivée de l'automation ont supplanté l'organisation tayloriste du travail, qui utilisait des travailleurs peu qualifiés et visait seulement à augmenter la cadence du travail. Aujourd'hui une formation générale de base, le recyclage professionnel et tout l'appareil de l'assistance qui garantit la santé physique et mentale sont indispensables, mais le soutien constant d'une famille dévouée est toujours utile, même dans les contextes les moins familiaux. De plus, le déclin

11. Dans le cas des variantes conservatrices, les circuits inadmissibles des dépenses publiques sont créés par les transferts d'argent aux familles, les systèmes de pensions, les mesures en faveur d'un plus grand soutien à l'offre de travail et les dépenses pour l'assistance (Esping-Andersen, 1996). 
des carrières professionnelles très productives dans le monde industriel a été largement compensé par l'accroissement de carrières semblables dans les services de pointe, les secteurs professionnels, la bureaucratie politique, l'administration des services d'assistance et, en particulier, dans les services financiers globalisés. Tout cela signifie que la sélection et le soutien aux formes d'emploi des moyens et des hauts salariés continue d'être une exigence de la régulation sociale et que seul un groupe minoritaire de travailleurs profitent de privilèges qui sont refusés aux autres.

Comme les familles nucléaires sont plus instables, représentent une part de plus en plus faible de la population et occupent une place de plus en plus restreinte dans le cycle de vie des gens, comme les emplois qui fournissent le revenu familial sont aussi de plus en plus rares, toutes les versions du régime du pourvoyeur sont de moins en moins efficaces en tant que mécanismes de redistribution économique et de modération des déséquilibres sociaux. Par ailleurs, les transformations actuelles créent des emplois peu productifs dans une proportion qui dépasse la capacité des différents systèmes de régulation de compenser ces situations sans consolider les zones qui sont socialement défavorisées de façon chronique. Ni l'extension massive des services publics, à la suédoise, ni la généralisation du travail précaire accompagné d'une très forte mobilité, comme aux État-Unis, ni l'adaptation novatrice, à l'italienne ou à l'allemande, des micro-entreprises et de la ségrégation domestique des femmes ne suffisent plus. La flexibilité, la précarité, les emplois à faible salaire constituent de plus en plus des traumatismes sérieux quand ils affectent les formes d'intégration sociale construites sur des équilibres fragiles dictés par la nécessité de soutenir les profils d'emplois qui jouissent de hauts salaires et de prestige social.

La recherche de la flexibilité accompagnée de formes d'intégration sociale a relancé l'autoemploi. En général, le dualisme des formes les plus intensives et les plus productives du travail salarié et les formes les plus extensives et flexibles du travail autonome peut persister comme mode de régulation là où il est possible de compenser le manque de productivité par des innovations qui exploitent la flexibilité. Mais certaines formes bénéfiques de complémentarité ne peuvent s'appliquer dans tous les contextes, car elles supposent l'existence de structures sociales et familiales stables, de type solidaire, et la possibilité technique d'augmenter la productivité de formes de travail plus discontinues à l'intérieur de l'organisation des micro-entreprises. Dans ce secteur, quelques entreprises artisanales et industrielles ont pu profiter de technologies qui peuvent s'adapter à la production à petite échelle (de l'électricité à la micro-électronique) et, dans certains contextes, de réseaux locaux d'organisation. Cependant, ces solutions ne s'appliquent pas au secteur tertiaire dans son ensemble, en particulier à celui des services de soins aux personnes qui utilisent du travail intensif peu qualifié. Ce ne sont pas là des carrières très enviables, d'autant qu'elles ne fournissent pas suffisamment de ressources matérielles et sociales pour survivre tout en s'intégrant à sa communauté d'appartenance. La prolongation du temps de travail et du temps de vie et l'utilisation la plus radicale de la communauté, de la solidarité familiale ou affinitaire ne suffisent pas à contrebalancer les contextes culturels et sociaux du travail peu productif avec des standards et des coûts de la vie élevés et des aspirations généralisées à la mobilité sociale ascendante. Pour toutes ces raisons, la vitalité du travail autonome n'est pas une solution applicable partout. Même dans le contexte du travail autonome et des petites entreprises, une cassure est en train de se dessiner entre d'un côté les circuits traditionnels, qui connaissent de plus en plus de difficultés et qui sont de moins en moins possibles pour les nouvelles générations socialisées avec les standards et les aspirations caractéristiques des contextes sociaux de haute productivité, et de l'autre côté les nouveaux circuits qui créent un équilibre entre la flexibilité et des conditions de vie acceptables mais qui ne s'étendent pas à tous les secteurs et ne peuvent pas s'installer dans des contextes marqués par la désintégration sociale.

Les transformations socio-démographiques contribuent aussi de différentes manières à la déstabilisation de la régulation sociale. En plus de la longévité, l'instabilité des mariages et les taux de fertilité bas sont en train de modifier l'interface entre soutien familial et carrières professionnelles vers une polarisation croissante entre des situations familiales plus stables et d'autres plus instables et socialement isolées. Non seulement la vie dans le cadre de familles nucléaires fondées sur le couple avec de jeunes enfants dépendants occupe-t-elle effectivement une part de plus en plus minime 
du cycle de l'existence, mais elle concerne une proportion toujours plus faible de la population et cela dans des conditions toujours plus hétérogènes. La chute du taux de fertilité affaiblit les réseaux de parenté, au détriment surtout de ceux dont la solidarité familiale constitue une ressource pour la micro-entreprise ou les activités d'assistance. On assiste simultanément à l'hétérogénéité grandissante des formes de familles, avec la multiplication des familles monoparentales, des familles reconstituées et des personnes seules, et à la polarisation entre familles qui vivent sur un salaire élevé ou sur plusieurs salaires et celles, qui souvent posent problème en termes de demande et de besoin d'aide, qui vivent d'un salaire bas et peu constant ou sont exclues du travail et contraintes d'avoir recours à l'assistance de l'État (Pahl, 1988a et 1988b). En d'autres termes, même si les exigences économiques de plus en plus nombreuses obligent un certain nombre de familles à compter sur plusieurs sources de revenus, une part importante de la population vit sur un seul bas salaire discontinu, ce qui se traduit par la paupérisation. De plus, Pahl insiste sur un autre aspect inquiétant de cette transformation. Les familles à salaires multiples développent toute une série de relations dans leurs milieux de travail qui leur sont utiles pour améliorer leurs situations professionnelles ou pour trouver du travail à leurs enfants ou à leur parentèle. Ce n'est cependant pas le cas des familles qui sont entretenues par des chômeurs ou des travailleurs précaires, qui ont pourtant bien plus besoin de telles ressources pour améliorer leur situation difficile (Morris, 1990, 1992). De plus, les perspectives d'autosuffisance sont envisageables seulement pour les familles à revenu moyen qui sont propriétaires de leurs maisons et qui sont bien intégrées à une structure sociale stable. Les familles à faible revenu dans des situations sociales plus dégradées sont exclues de ces formes modernisées de l'auto-approvisionnement. Dans ces conditions, la cassure entre les familles « riches en travail » et les familles « pauvres en travail » devient véritablement un circuit de polarisation sociale (Pahl, 1988b).

\section{DÉSTABILISER LA QUESTION SOCIALE VERS LA PAUVRETÉ URBAINE ET L'EXCLUSION SOCIALE}

Les transformations de l'emploi des quelques dernières décennies ont été diversement interprétées par les sciences sociales comme des phénomènes contingents requérant une adaptation sociale aux lois d'airain du marché ou comme un changement d'époque pour le marché qui en serait désormais à l'étape mythique de la «globalisation». Ce sont les débats sur les emplois et activités informels, donc sur la formation d'une underclass, et sur l'exclusion sociale qui ont désigné la régulation du travail et la question sociale comme les zones critiques des transformations en cours.

Le débat sur le travail informel ${ }^{12}$ a contribué à souligner quelques aspects des difficultés croissantes qui affectent les systèmes de régulation des régimes d'emploi et des régimes sociaux. L'informel est presque toujours un signe de crise de la régulation sociale. Ce n'est que rarement qu'il a entraîné la création de formes d'emplois flexibles compatibles avec les stratégies d'intégration sociale de la population. Même les exemples les plus saisissants d'activités multiples ou d'autoapprovisionnement montrent à quel point il est important de trouver des sources supplémentaires de revenu pour arriver à bonifier l'éventail des ressources insuffisantes garanties par la complémentarité entre revenus de travail, activités non-payées et services d'assistance assurés par l'État. Cependant, ce sont surtout les formes de travail au noir, précaires et temporaires qui montrent la divergence entre d'une part les activités de travail et d'autre part les conditions minimales de vie et

12. Le débat sur les activités informelles dans les pays industrialisés connurent une phase d'intensité dans les années 1980 qui déclina pour s'éteindre tout à fait ces dernières années. Le terme «informel » fut utilisé pour la première fois par HART (1973) dans une étude effectuée pour le Bureau international du travail sur l'économie urbaine d'une ville africaine (Accra au Ghana) caractérisée par la persistance d'un mélange d'activités de subsistance et de petits emplois dans le marché. Autour de la fin des années 1970, le terme fut importé vers les pays industrialisés pour parler non plus d'un secteur ou d'une localité particulière, mais pour désigner des activités et des formes de travail qui échappent au contrôle de l'État parce qu'elles ne sont pas identifiées, enregistrées et taxées (comme les emplois occasionnels ou l'auto-approvisionnement) ou entreprises à l'encontre des réglementations en vigueur (comme les multiples activités non déclarées, le travail des employés sans contrat, sans assurances et sans taxations) (Ferman et al., 1978 ; Ferman et Berndt, 1981 ; Pahl, 1984). 
d'intégration sociale. L'emploi précaire et le travail informel témoignent de ce que les conditions de travail sont en dessous du standard général de vie socialement nécessaire pour s'intégrer normalement dans sa communauté d'appartenance. Si ces conditions persistent, sont chroniques et subies par les individus et les groupes qui connaissent aussi une discrimination de la part des institutions, il devient très difficile de rester attaché aux horizons, aux valeurs, aux perspectives et aux comportements typiques de sa propre communauté d'appartenance.

C'est précisément en ce sens que le débat sur l'underclass ${ }^{13}$ et sur l'exclusion sociale a mis l'accent sur le fait que la transformation des régimes d'emploi n'a pas seulement créé des tensions dans le système de régulation, mais qu'elle trouve également son expression dans des formes de désintégration sociale qu'il est difficile de briser. Le cumul des handicaps au niveau social et au niveau de l'emploi parmi certains groupes de la population - minorités, immigrants récents, habitants de quartiers pauvres dans certaines zones urbaines des régions les plus touchées par la désindustrialisation et caractérisées par des formes plus faibles ou dépendantes d'industrialisation — se change en véritable syndrome d'exclusion sociale de masse, comprise comme une forme chronique et institutionnalisée de refus à l'accès aux possibilités et aux ressources qui garantissent les standards de vie de la majeure partie de la population des pays industriels avancés (Castel, 1995a et 1995b). Ce débat, aux États-Unis sur l'underclass et en Europe sur l'exclusion sociale, attire l'attention sur l'émergence de ces syndromes, qui sont avant tout des conséquences de la crise des formes de régulation adoptées par les régimes d'emploi et les régimes sociaux tels qu'ils se développèrent pendant le cycle historique intensif, en particulier au dernier stade, le plus marqué par le système de l'État-providence.

La déstabilisation de la question sociale est compliquée par son occurrence sur deux fronts très intimement reliés. La plus grande instabilité et l'hétérogénéité des carrières professionnelles et des structures des foyers ouvrent une vaste zone où le risque de manque d'intégration sociale s'accroît. Ceci est l'effet majeur commun à tous les modèles de sociétés industrielles avancées dans la transition fordiste. La protection assurée par l'État, les familles et les ressources du marché, dans ses diverses formes structurées par une croissance intensive, n'est plus apte à procurer une couverture totale aux sujets qui vivent avec des carrières professionnelles instables ou faiblement rémunérées ou à ceux qui, de plus en plus nombreux, finissent par vivre seuls ou dans des familles accablées de problèmes et mal équipées pour y faire face.

La zone du besoin et du mouvement de marginalisation est en train de s'élargir et de briser le cycle de la croissance et du contrôle économique de la question sociale qui suit les lignes de division et de sélection typiques de la période des Trente Glorieuses de l'après-guerre. Au cours de cette période, on est arrivé à contrôler la question sociale non pas parce qu'il n'existait pas de situation de malaise ni de sérieuses difficultés, mais grâce à l'efficacité de l'ensemble diversifié des combinaisons de trois facteurs. D'abord, il y eut la capacité d'intervention du système d'assistance public, particulièrement développé dans les pays scandinaves, mais aussi dans une certaine mesure ailleurs ${ }^{14}$. La mobilité sociale à grande échelle empêcha alors qu'un nombre considérable de sujets aient à vivre de longues périodes de difficultés ; ce fut plus répandu dans les modèles de laissezfaire mais aussi dans les autres modèles, surtout en termes de mobilité sociale masculine ascendante. Enfin, des familles et des communautés relativement normales étaient capables de fournir un

13. Le terme underclass a été employé aux États-Unis pour désigner surtout les conditions de vie des Afro-Américains concentrés dans les ghettos des grandes villes (Wilson, 1987 et 1993), tandis que l'expression exclusion sociale est apparue plus récemment, même dans le langage officiel, dans les pays de l'Union européenne (Castel 1995a ; Silver,1996). Voir le premier et le dernier chapitre de MINGIONE (1996) pour un débat sur le lien entre les deux concepts d'underclass et d'exclusion sociale et pour des références à des ouvrages sur la question.

14. Il est peut-être utile de rappeler à cet égard la « guerre » contre la pauvreté menée par l'administration Johnson le gouvernement américain considéré comme le chef de file du modèle du laissez-faire — pendant la seconde moitié des années soixante. Bien qu'à partir de la seconde moitié des années soixante dix la proportion de la population vivant sous le seuil de la pauvreté selon le Federal Poverty Standard ait recommencé à monter, atteignant des taux à peine inférieurs à ceux de 1960 quelques années auparavant, les résultats obtenus dans les dix premières années d'intervention furent substantiels et la teneur « providentialiste » du programme ne fait aucun doute. 
réseau adéquat de première sécurité aux sujets en difficulté ${ }^{15}$. L'effondrement financier des programmes d'assistance étatiques et les tendances à la déréglementation du marché du travail sont des indicateurs de ce processus. Mais la croissance tendancielle ${ }^{16}$ de la proportion de la population vivant au-dessous du seuil de la pauvreté révèle l'ampleur du problème et trouve une expression dans les convulsions sociales.

C'est dans ce processus plus général d'un risque accru de marginalisation et d'un déficit d'intégration que se pose la question de l'exclusion sociale. Pour des raisons liées à l'environnement (ghettos ou quartiers délabrés des grandes villes touchées par la désindustrialisation), à l'origine sociale (minorités défavorisées, immigrants, gitans) ou à l'aboutissement d'un parcours d'appauvrissement plus sérieux et cumulatif, une partie de la population marginalisée se retrouve victime de ségrégation, dans un état de privation tel qu'il finit par coïncider avec une véritable discrimination institutionnelle sans qu'un retour à la normale soit vraisemblable, état qui impose des conditions de vie et des formes de comportement stigmatisées par le reste de la population. Dans cette perspective, la description de Wilson (1987 et 1993) sur les pauvres des ghettos et celle de Castel (1995a et 1995b) sur l'exclusion sociale ${ }^{17}$ vont tout à fait dans le même sens, même si les proportions et certaines caractéristiques du phénomène sont différentes aux États-Unis et en Europe continentale.

La déstabilisation de la question sociale provoque partout des tensions qui à la fois élargissent la zone de risque et activent les problèmes d'exclusion sociale chez les population défavorisées, surtout si elles sont confinées dans les banlieues délabrées des grandes villes. Comme l'ont noté à la fois Wilson et Sen, la ségrégation territoriale finit par être en un facteur de risque et conduit aisément à une exclusion sociale chronique. Dans les ghettos et les quartiers pauvres, l'accès à des perpectives d'emplois stables est difficile en raison de la discrimination ; les services d'assistance sont inadéquats et de piètre qualité et l'on doit adopter des formes particulières de comportement pour faire face aux difficultés de tous les jours. S'amorce donc un cercle vicieux qui combine la mise en ghettos d'une partie des pauvres et la discrimination institutionnelle qui transforme les syndromes de la pauvreté économique en exclusion sociale. Le cercle vicieux est activé par le fait que les perspectives d'échapper à la pauvreté sont encore réduites par la ségrégation et donc par l'efficacité particulière de la discrimination négative. Cet itinéraire a été largement exploré et débattu dans le cas des pauvres des ghettos aux États-Unis, à partir de la contribution de Wilson dans The Truly Disadvantaged (1987). Dans les autres pays industrialisés, le problème n'est ni aussi manifeste, ni aussi étudié. Cette différence tient aux divers modèles d'assistance, qui sont caractérisés par des interventions plus effectives de l'État, une moindre exposition aux forces du marché et, dans certains cas, par une capacité plus grande à protéger la famille et la communauté.

En conclusion, examinons rapidement les significations, les formes et les limites des mesures de prévention adoptées pour éviter la propagation de l'exclusion sociale dans d'autres contextes urbains ${ }^{18}$. L'analyse comparée menée par Wacquant (1996) dans un ghetto de Chicago et dans la cité de La Courneuve donne des pistes qui devraient être développées par des études longitudinales de communautés défavorisées et victimes de ségrégation dans les grandes villes européennes. Même si elle s'accompagne de divisions ethniques et de discrimination contre les immigrants ou les

15. Ici la spécificité des variantes familiales — y compris aussi quelques minorités ethniques aux États-Unis — tient au fait que des groupes affinitaires importants et stables bien établis sur le territoire, fournissent des réseaux communautaires et d'affinités avec des ressources financières et humaines nouvelles et allant bien au-delà de l'intervention de première ligne.

16. En réalité, il s'agit d'une tendance sur une longue période et on ne peut donc référer à de courtes séquences de données quitte à tenir compte des fluctuations à court terme. De telles opérations débouchent sur des données quelque peu manipulées se prêtant à des interprétations diverses mais, quoi qu'il en soit, on constate une tendance à la hausse préoccupante dans tous les pays, beaucoup plus évidente et substantielle dans les zones de laissez-faire (surtout aux États-Unis et au Royaume-Uni) et qui commence à se faire sentir maintenant dans les social-démocraties.

17. Dans sa définition analytique de l'exclusion sociale, Robert CASTEL (1995b, p. 19) insiste particulièrement sur le cumul de la discrimination négative et du statut institutionnel. « [...] l'exclusion [...] est ainsi toujours l'aboutissement de procédures officielles et représente un véritable statut. C'est une forme de discrimination négative qui obéit à de strictes règles de construction. »

18. Pour des références plus détaillées à ce débat voir MINGIONE (1996, pp. 379-83). 
minorités, la ségrégation est ici moins étouffante que dans bien des villes américaines ${ }^{19}$. Cela est dû aux diverses combinaisons des traditions «providentialistes » (plus importantes dans les pays scandinaves et au Royaume-Uni) et des traditions de solidarité communautaire relativement ouvertes fournies par des ressources de protection, surtout parce que les systèmes familiaux résistent mieux aux tendances déstabilisantes de la transition démographique. On peut observer le même phénomène dans les villes américaines si l'on compare les minorités jouissant de moins de ressources communautaires, les Afro-Américains et les Porto-Ricains, avec d'autres minorités qui ont des systèmes familiaux plus traditionnels (Moore et Pinderhughes, 1993). Le problème, c'est que ces combinaisons qui sont plus ouvertes, et donc moins orientées vers la discrimination institutionnelle, constituent des formes de résistance «conservatrices » plutôt que des stratégies nouvelles pour construire de nouveaux équilibres d'intégration sociale qui seraient appropriés au caractère flexible et fragmentaire du cycle historique que nous vivons aujourd'hui. En tant que telles, elles ont leur prix et leurs limites : l'effondrement des dépenses publiques, l'impossibilité de contrôler la hausse du taux de chômage, les difficultés croissantes du système familial et l'interaction de plus en plus inégalitaire et discriminatoire entre ceux qui peuvent compter sur une famille capable de les aider et ceux qui ne le peuvent pas. En bref, la différence entre les États-Unis et les autres pays semble résider dans le fait que dans les villes américaines, la déstabilisation du social a des effets immédiats ${ }^{20}$, tandis que partout ailleurs, il s'agit d'un processus de lente érosion.

Cet article a amorcé l'analyse de l'impact des changements qui ont cours depuis presque vingt ans en termes de liens entre les nouveaux régimes économiques et démographiques et les facteurs sociaux qui engendrent la coopération et les chaînes de socialité. Vient de s'achever une longue phase qui a vu la prédominance des organisations associatives, des économies d'échelle, de la standardisation et de la bureaucratisation et du monopole de la régulation par les États nations. Cependant, la transition n'a pas encore clairement marqué les nouveaux équilibres entre les perspectives économiques et les modes d'intégration sociale. Dans tous les modèles de capitalisme «providentialiste », les transformations produisent des tendances à la désagrégation. Au-delà d'un apparent succès économique immédiat - comme au Japon, dans certaines régions d'Italie, en Corée et, plus récemment en Hollande - à moyen terme, ces tendances empêchent toute augmentation du bien-être et de la solidarité, de la stabilité sociale et de nouvelles formes de coopération. La recherche sociologique à ce propos peut aller au-delà du paradigme du marché et souligner l'importance des mélanges de facteurs associatifs et de nouveaux réseaux de réciprocité locaux et étendus à la communauté entière pour le maintien du contrôle des itinéraires familiaux et professionnels les moins stables et les plus hétérogènes et pour éviter la formation de zones d'exclusion sociale. C'est là sans aucun doute l'un des champs de bataille du « second souffle de la sociologie ».

\section{Enzo MINGIONE Département de Sociologie Université of Padoue Fondazione Bignaschi \\ Via Olmetto 3 20123 Milan, Italie}

\section{RÉSUMÉ}

Cet article est fondé sur une critique radicale du paradigme du marché auto-régulé et facteur de régulation sociale. On soutiendra que les relations d'échange ne peuvent avoir lieu que dans des conditions favorables que ne peut pas directement créer le marché lui-même, mais qui sont plutôt imputables à des facteurs de coopération (association et réciprocité) constituant la base de l'enracinement social de l'économie. Ceci a deux conséquences importantes : d'une part, les différentes conditions sociales (dans le temps et dans l'espace) sont essentielles à la compréhension des modes de développement industriel ; d'autre part, les

19. Ralph DAHRENDORF (1992, p. 556) souligne cette plus grande ouverture en termes d'opportunités, de modèles culturels et de services d'assistance qui favorise la mobilité ascendante également dans le cas des villes du Royaume-Uni.

20. À ce propos, on peut noter un parallèle historique significatif dans l'explosion de la pauvreté urbaine dans les villes anglaises de la seconde moitié du siècle dernier, un effet de la crise dans le cycle extensif de l'accumulation qui conduira aux impulsions organisées en faveur de l'État-providence du cycle intensif. 
sociétés industrielles se caractérisent par des différences importantes et persistantes. L'époque fordiste a été caractérisée par un mélange de conditions où les associations étaient prédominantes tandis que l'époque actuelle se caractérise par un retour de la réciprocité ; c'est en ce sens que l'on considère que les sociétés contemporaines sont fragmentées. On fera ici une étude comparative montrant à quel point les traits de la fragmentation se manifestent dans la production de l'exclusion sociale, c'està-dire d'un statut discriminatoire appliqué institutionnellement, venant s'ajouter à certains processus de marginalisation tenant au chômage et à la transformation des systèmes socio-démographiques et des systèmes d'emploi.

\section{SUMMARY}

This paper is based on a radical critique of the paradigm of the self-regulated and socially regulating market. It is argued that exchange relations can take place only within favourable conditions which cannot be directly created by the market itself but rather by factors of cooperation (association and reciprocity) which constitute the basis for the social embeddedness of the economy. This fact has two important consequences: the differing (in time and space) socio-cultural conditions are essential for understanding the modes of industrial development; industrial societies are characterized by persistent and important differences. The Fordist age was characterized by mixes in which the associative forms were predominant while now the combinations are characterized by the innovative resurgence of reciprocity; in this sense the present societies are considered as fragmented. A comparative investigation is carried out of the extent to which the features of fragmentation are reflected in the production of social exclusion, that is institutionally enforced discriminated status, on top of marginalization processes, particularly centred on joblessness and the transformation of the employment and socio-demographic systems.

\section{RESUMEN}

Este artículo está fundado en una crítica radical del paradigma del mercado autoregulado y del factor de regulación social. Se sostendrá que las relaciones de intercambio no pueden tener lugar sin condiciones favorables que no pueden ser creadas por el mismo mercado, sino por factores de cooperación (asociación y reciprocidad) que constituyen la base del arraigo social de la economía. Esto tiene dos consecuencias importantes : por un lado, las diferentes condiciones sociales (diferentes en el tiempo y en el espacio) son esenciales para la comprensión de los modos de desarrollo industrial; y por otro lado, las sociedades industriales se caracterizan por diferencias importantes y persistentes. La época fordista se caracterizó por una mezcla de condiciones en el marco de las cuales las asociaciones eran predominantes mientras que la época actual se caracteriza por un retorno de la reciprocidad ; es en este sentido que se considera que las sociedades contemporáneas están fragmentadas. Haremos aquí un estudio comparativo que muestra hasta que punto los rasgos de la fragmentación se manifiestan en la producción de la exclusión social, es decir de un estatuto discriminatorio aplicado institucionalmente, que viene a agregarse a ciertos procesos de marginalización y particularmente centrada en el desempleo y la transformación de los sistemas socio-demográficos y de los sistemas de empleo.

\section{BIBLIOGRAPHIE}

ARRIGHI, Giovanni (1994), The Long Twentieth Century, Londres, Verso.

BENOÎT-GUILBOT, Odile et GALLIE Duncan (dir.) (1992), Chômeurs de longue durée, Arles, Actes Sud.

BRAUDEL, Fernand (1977), Afterthoughts on Material Civilization and Capitalism, Baltimore, MD, Johns Hopkins University Press.

BRAUDEL, Fernand (1986a), Les Structures du quotidien, Paris, Armand Colin.

BRAUDEL, Fernand (1986b), Le Temps du monde, Paris, Armand Colin.

CASTEL, Robert (1995a), Métamorphose de la question sociale. Une chronique du salariat, Paris, Fayard.

CASTEL, Robert (1995b), «Les pièges de l'exclusion », Lien social et politique. RIAC, nº 34, pp. 13-21.

CASTELLS, Manuel (1996), The Rise of Network Society, Oxford, Basil Blackwell.

D'IRIBARNE, Philippe (1990), Le Chômage paradoxal, Paris, PUF.

DAHRENDORF, Ralph (1992), Il Conflitto Sociale nella Modernità, Bari, Laterza.

ESPING-ANDERSEN, Gøsta (1990), The Three Worlds of Welfare Capitalism, Cambridge, Polity Press.

ESPING-ANDERSEN, Gøsta (1995), «Il welfare state senza il lavoro. L'ascesa del familismo nelle politiche sociali dell'Europa continentale », Stato e Mercato, n ${ }^{\circ} 45$, pp. 347-380.

ESPING-ANDERSEN, Gøsta (dir.) (1996), Welfare States in Transition: National Adaptations in Global Economies, Londres, Sage.

FERMAN, L.A., BERNDT, L. et SELO, E. (1978), « Analysis of the Irregular Economy : Cash Flow in the Informal Sector », unpublished report of the Bureau of Employment and Training, Michigan Department of Labour and Industrial Relations, Ann Arbor, Michigan, Wayne State University.

FERMAN, L.A. et BERNDT, L. (1981), «The Irregular Economy », in S. Henry (dir.), Can I Have it in Cash ?, Londres, Astragel Books, pp. 26-42.

GERSHUNY, Jonathan (1978), After Industrial Society : The Emerging Self-Service Economy, Londres, Macmillan.

GERSHUNY, Jonathan (1983), Social Innovation and the Division of Labour, Oxford, Oxford University Press.

GERSHUNY, Jonathan et MILES Ian (1983), The New Service Economy: The Transformation of Employment in Industrial Societies, New York, Praeger.

GRANOVETTER, Mark (1985), «Economic Action and Social Structure: The Problem of Embeddedness », American Journal of Sociology, $91, \mathrm{n}^{\mathrm{o}} 3$, pp. 481-510. 
HART, K. (1973), « Informal Income Opportunities and Urban Employment in Ghana », Journal of Modern African Studies, $\mathrm{n}^{\mathrm{o}} 11, \mathrm{pp} .61-89$.

LESTHAEGHE, Ron (1991), « The Second Demographic Transition in Western Countries : An Interpretation », IPD working paper, $\mathrm{n}^{\mathrm{o}} 2$, Bruxelles, Vrjie Universiteit.

MALINVAUD, Edmond (1984), Mass Unemployment, Oxford, Blackwell.

MILES, Ian (1983), « Adaptation to Unemployment », Spru Occasional Paper, $\mathrm{n}^{\circ} 20$

MINGIONE, Enzo (1991), Fragmented Societies, Oxford, Blackwell.

MINGIONE, Enzo (dir.) (1996), Urban Poverty and the Underclass : A Reader, Oxford, Blackwell.

MOORE, Joan et PINDERHUGHES, Raquel (dir.) (1993), In the Barrios. Latinos and the Underclass Debate, New York, Russell Sage Foundation.

MORRIS, Lydia (1990), The Workings of the Household : a US-UK Comparison, Cambridge, Polity Press.

MORRIS, Lydia (1992), « The Social Segregation of the Long Term Unemployed», Sociological Review, n 38, pp. 344-369.

PAHL, Raymond (1984), Divisions of Labour, Oxford, Blackwell.

PAHL, Raymond (1988a), On Work : Historical, Comparative and Theoretical Approaches, Oxford, Blackwell.

PAHL, Raymond (1988b), « Some Remarks on Informal Work, Social Polarisation and the Social Structure », International Journal of Urban and Regional Research, vol. 12, $\mathrm{n}^{\circ} 2$, pp. 247-267.

PIRENNE, Henri (1953), «Stages in the Social History of Capitalism» in R. Bendix et S. Lipset (dir.), Class, Status and Power : A Reader in Social Stratification, Glencoe, IL, The Free Press, pp. 501-517.

POLANYI, Karl (1983), La Grande Transformation : aux origines politiques et économiques de notre temps, Paris, Gallimard. PUGLIESE, Enrico (1993), Sociologia della Disoccupazione, Bologne, Il Mulino.

REYNERI, Emilio (1996), Sociologia del Mercato del Lavoro, Bologne, Il Mulino.

RIFKIN, Jeremy (1996), La Fin du travail, Montréal, Boréal.

SASSEN, Saskia (1991), La Ville globale : New York, Londres, Tokyo, Paris, Descartes \& Cie.

SASSEN, Saskia (1996), « Service Employment Regime and the New Inequality » in E. Mingione (dir.), op. cit., pp. 64-82.

SILVER, Hilary (1996), «Culture, Politics and National Discourses of the New Urban Poverty », in E. Mingione (dir.), op. cit., pp. 105-138.

WACQUANT, Loï (1996), «Red Belt, Black Belt : Racial Division, Class Inequality and the State in the French Urban periphery and the American Ghetto », in E. Mingione (dir.), op. cit., pp. 234-274.

WALLERSTEIN, Immanuel (1995), Impenser la science sociale : pour sortir du XIX ${ }^{e}$ siècle, Paris, PUF

WALLERSTEIN, Immanuel (1996), Le Capitalisme historique, Paris, Desclée De Brouwer.

WILSON, William Julius (1987), The Truly Disadvantaged: The Inner City, the Underclass, and Public Policy, Chicago, University of Chicago Press.

WILSON, William Julius (dir.) (1993), The Ghetto Underclass, Londres, Sage. 